\title{
Review
}

\section{Multimorbidity and Frailty: Tackling Complexity in Parkinson's Disease}

\author{
Emma Tenison $^{\mathrm{a}}$ and Emily J. Henderson ${ }^{\mathrm{a}, \mathrm{b}, *}$ \\ ${ }^{a}$ Department of Population Health Sciences, Bristol Medical School, University of Bristol, Bristol, \\ United Kingdom \\ ${ }^{\mathrm{b}}$ Older People's Unit, Royal United Hospitals Bath NHS Foundation Trust, Combe Park, Bath, United Kingdom
}

Accepted 28 June 2020

\begin{abstract}
Parkinson's disease (PD) is a condition that predominantly affects older people. It is imperative that clinical management considers the other significant illnesses that people with PD accumulate as they age in conjunction with their resilience to cope with physiological change. Multimorbidity and frailty act synergistically to heighten the risk of adverse outcomes for older people with PD. These states are associated with increased likelihood of hospitalization, polypharmacy, adverse drug effects including the anticholinergic burden of medications, drug-disease and drug-drug interactions. Management should be integrated, holistic and individualised to meticulously balance the risks of interventions considering the vulnerability of the individual to recover from disturbance to their environmental, physical and cognitive equilibrium.
\end{abstract}

Keywords: Aging, comorbidity, frailty, integrated care, multimorbidity, Parkinson's disease, polypharmacy

\section{INTRODUCTION}

Parkinson's disease (PD) is rare before age 50 and, whilst it is not a result of ageing per se, increased age remains the strongest risk factor for development [1]. Prevalence rises steeply with age from $1 \%$ of all those over 60 years up to $4 \%$ in those over 85 years [1]. Ageing influences PD progression with older people experiencing a faster rate of motor decline and reduced levodopa responsiveness [2].

In older adults and as PD progresses, a single 'disease' framework for providing care is often inadequate and management needs to consider the distinct but overlapping concepts of multimorbidity and frailty. The synergy between PD, multimorbidity

\footnotetext{
${ }^{*}$ Correspondence to: Dr Emily J. Henderson, Department of Population Health Sciences, Bristol Medical School, University of Bristol, Bristol, BS8 1NU, United Kingdom. Tel.: +44 01225 821028; E-mail: Emily.Henderson@bristol.ac.uk.
}

and frailty drives clinical complexity [3]. Furthermore, comorbid conditions and/or inclusion criteria with upper age limits [4] lead to older people with PD being under-represented in research [5], introduce significant bias and limit the generalisability of findings.

This paper aims to define frailty, multimorbidity, and related concepts; and describe their relationship with PD. We explore the implications for care in terms of prescribing and the risks of hospitalization as stressors that can precipitate decline in this vulnerable group. Ultimately, physiological ageing, declining resilience to stressors, disease heterogeneity, and polypharmacy converge to yield a cluster of scenarios which are highly prevalent and interdependent. Proactive, coordinated, and holistic care is imperative to avoid decompensation of the fragile equilibrium that frequently precipitates irreversible loss of function and catastrophic harm though prescribing and hospitalization. 


\section{CONCEPTS}

The terms comorbidity, mutimorbidity, and frailty are related but distinct, describing states that confer excess risk. Comorbidity is the presence of additional diseases in relation to an index disease, in one individual [6]. Comorbidity can be quantified by means of numerical disease counts; weighted index measures; patterns of disease association based on statistical association or expert consensus; or the speed of disease accumulation [6]. The weighted Charlson Comorbidity Index is reliable [7] and frequently utilised in PD studies ([7-10]. Multimorbidity is an evolution of the concept comorbidity and was made distinct from the MeSH term 'comorbidity' in 2018. Multimorbidity is defined as the manifestation of two or more chronic diseases in the same person [11]. Despite not attributing weight to different illnesses [12] the impact of multimorbidity on an individual's risk profile may be greater than the sum of conditions [12]. Synergy between conditions results in steeper deterioration in function driven in part by an imbalance between the indirect and direct effect of one illness on another as well as drug-disease interactions [13].

Frailty, originally a geriatric medicine concept which has since been embraced by multiple medical and surgical specialties, describes a multidimensional, dynamic state [14]. It is broadly recognised as a syndrome of loss of physiological reserve which confers greater vulnerability to negative health outcomes and which has been described both in terms of a frailty phenotype (FP) model, as well as a frailty index (FI) based on accumulation of deficits [14]. Sarcopenia, a disease characterised by low muscle strength, together with low muscle quantity or quality, contributes to the development of physical frailty [15] Frailty and sarcopenia overlap since the FP model includes reduced grip strength, a feature of sarcopenia, and also reduced gait speed which indicates poor physical performance and categorises sarcopenia as severe. Risk factors for onset of frailty can be grouped into sociodemographic, clinical, lifestyle and biological domains [14]. Some specific factors, such as physical inactivity and malnutrition, may be potentially modifiable with interventions, whilst the risk of polypharmacy, commonly defined as five or more different types of medication, must be carefully balanced against potential benefits of medication [14]. Frailty is associated with increased falls, institutionalisation and mortality, underlining the importance of strategies to delay the onset or progression of frailty [14].

\section{COMORBIDITY AND PD}

Numerous studies have focussed on the association between PD and single other conditions. In early disease the burden of comorbidity tends to be low. In the ICICLE cohort, coding conditions using the International Classification of Primary Care-2 (ICPC-2) that tend to overlap less with non-motor symptoms, did not demonstrate a difference in number of conditions between the PD and control groups [8] which is consistent with other studies [7]. A large crosssectional study using a Scottish primary care dataset showed that, after adjusting for age, sex and deprivation, PD patients had a greater number of both physical and mental health comorbidities compared to controls. Mental health conditions were particularly prevalent $(44 \%)$ which is consistent with the well-recognised neuropsychiatric manifestations of PD [16] and $31 \%(817 / 2640)$ had 5 or more conditions [10]. A smaller prospective cohort $(n=147$ PD patients) found a higher number of comorbidities in PD patients than in controls and noted an accumulation of comorbidity over 3 years [9]. Comorbidity predicted mortality after correcting for age, disease duration/stage, motor and non-motor symptoms (Hazard ratio 1.285 (95\% confidence interval, 1.047-1.577; $p=0.017)$ ) [9]. People with PD have more causes of death cited on death certificates than decedents of similar age with dementia and pneumonia being common contributors to death [17].

By nature of the concept, few studies address multimorbidity and PD in isolation. In one PD-specific study, the presence of multimorbidity exerted only a small negative effect on quality of life. These findings may reflect the minimal impact of multimorbidity at a relatively early disease stage or insensitivity of the implicit lack of weighting associated with dichotomising multimorbidity into present/absent [8]. In an Italian primary care cohort of 3189 multimorbid patients, those with PD showed the greatest difficulty with self-care and performing usual activities. The diagnosis of PD had the greatest impact on quality of life of all 45 conditions studied [18]. Cluster analyses have sought to determine the co-occurrence of conditions beyond chance. The Octabaix crosssectional study examined a community sample of people aged 85 and identified four clusters from 
16 index conditions. People with PD had an average of 4.46 (SD 1.45) comorbidities and a cluster of conditions was identified comprising dementia, PD, dyslipidaemia, peripheral arterial disease and anaemia [19]. The association between PD and cardiovascular morbidity may be mediated by activity levels, gender, smoking and potentially diabetes [20] and anaemia is a putative risk factor for PD [21].

\section{FRAILTY AND PD}

The majority of studies examining frailty in PD have utilised the phenotypical approach albeit operationalised in a variety of ways and sampled from a general population using cross-sectional design [22]. The wide prevalence of frailty in PD across studies (29-67\%) is a likely function of the varying measures employed. In two small studies, frailty was more prevalent in those with more advanced PD $[23,24]$ and is associated with disability [25]. The COMPASS-ND study examined prevalence of frailty, calculated using the FI and FP models, across neurodegenerative disorders [26]. Prevalence of frailty across all ten conditions was similar: $11 \%$ (FI) and $14 \%$ (FP) and the low rates likely represent a clinical trial sample not entirely representative of the normal population [26]. The inclusion of very small numbers of patients with PD and related disorders $(n=24$ in total) make it difficult to draw conclusions except to note that use of both FP and FI were feasible and yielded similar prevalence rates [26]. In a cross-sectional community-based study, frailty was associated with cognitive impairment, hallucinations and dementia independent of age, sex and comorbidities [23]. In hospitalised patients with PD it is a useful prognostic marker. The Clinical Frailty Scale (CFS) quantifies frailty on a 9-item scale with higher scores reflecting higher frailty. In patients aged over 75 years, admitted to a UK hospital with PD over 2 years, 84\% (330/393) were significantly frail (CFS scores 5-9). Despite controlling for age, sex, comorbidities and neuropsychiatric syndromes, those with the most severe degrees of frailty (CFS 7-9) had an 8 fold increased odds of dying in hospital [27].

The phenotypical definition of frailty embeds parameters of physical function [28] which contributes to the degree of overlap between the features of PD and frailty and which could over-diagnose frailty amongst people with PD [26]. The cumulative deficit model of frailty used in the COMPASS-ND study excluded items relating to neurological disease, such as parkinsonism and, whilst many of the items in the index are recognised non-motor symptoms of PD, their presence is likely to reflect an individual's health status, whether or not they are a direct result of the PD itself [26]. Additionally, sarcopenia is common amongst people with PD [29, 30] so may therefore be a key component of the pathway leading from PD symptoms to frailty and disability [15] and itself may partly be triggered by malnutrition, which affects up to $24 \%$ of people with PD [31].

Whilst no instruments have been validated specifically in PD, studies using both FI and FP derived approaches have shown associations with negative outcomes [32, 33]. Most of the studies that have examined frailty in PD have used phenotype criteria [34]. The index-based approach may over-estimate frailty given the contribution of multiple motor and non-motor symptoms to the scoring whereby neither the aetiology nor severity of each deficit is considered. When this has been operationalised it has been suggested that health status is affected regardless of the aetiology of the deficit [26]. Nonetheless, future efforts may focus on aligning measures to specific negative sequelae and quantifying the degree of physiological dysregulation from each PD symptom and the extent therefore to which this renders homeostasis vulnerable to irretrievable disruption with specific stressors.

\section{IMPLICATIONS FOR CLINICAL PRACTICE: PRESCRIBING AND HOSPITALIZATION}

Older adults have a high risk of adverse drug events secondary to altered pharmacokinetics and pharmacodynamics. Polypharmacy increases the risk of drug-drug and drug-disease interactions and is frequently an issue in PD due to the complexity of the disease and its treatment. Close medication review is a key part of the National Institute of Health and Care Excellence management of people with multimorbidity [35]. In a specialist movement disorder centre, patients with on average 10-year disease duration, at least one comorbidity and 5 or more drugs, showed frequent potential interactions between their PD medication and co-prescribed medications. These included interactions between CNS drugs and dopaminergic therapy, co-prescription of QT prolonging drugs particularly with apomorphine, and interactions between P450 metabolised drugs, ropinirole and entacapone [36]. $73.3 \%$ of people with 


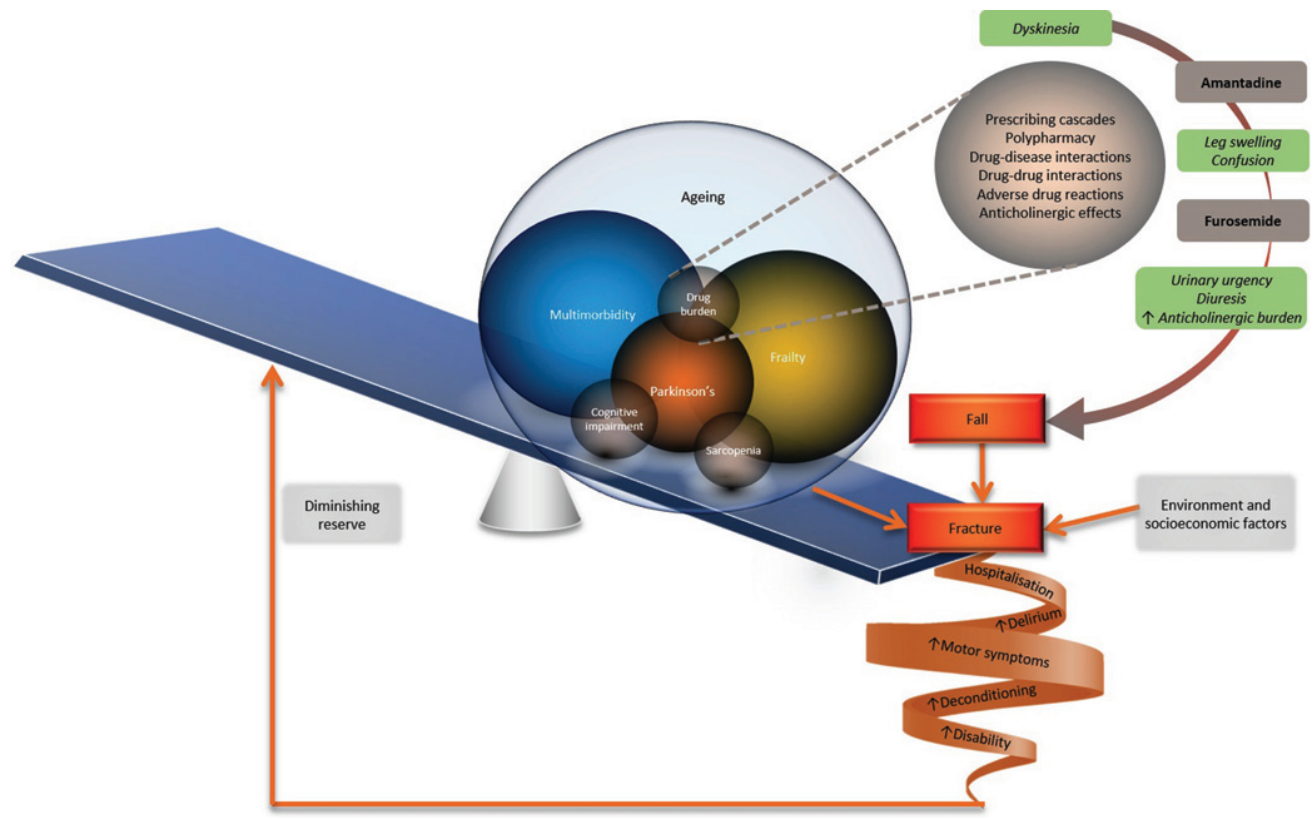

Fig. 1. The impact of multimorbidity and frailty in Parkinson's disease. Many individuals with Parkinson's disease will experience the disease in the context of multimorbidity and/or be living with frailty. In these circumstances, relatively small physiological insults 'tip the balance' precipitating decompensation of a fragile equilibrium. Prescribing cascades, whereby ever-increasing number of drugs are added to ameliorate adverse effects of other drugs, can add to polypharmacy and precipitate adverse events such as falls and fractures. This frequently necessitates hospitalization resulting in experience of delirium, deconditioning and worsening disability. Already diminished physiological reserve and resilience are further eroded, fuelling the vicious cycle and negatively impacting quality of life. (C) Tenison/Henderson.

Table 1

Take home messages

- Older people with Parkinson's disease experience a faster rate of motor progression, together with earlier occurrence of falls and cognitive impairment.

- Multimorbidity, frailty and Parkinson's disease frequently coexist and lead to clinical complexity, which can be challenging to manage within existing healthcare systems.

- Increasing comorbidity or frailty, alongside Parkinson's disease, increases negative outcomes, including mortality.

- The complexity of Parkinson's disease frequently leads to prescribing cascades and polypharmacy which can trigger interactions, worsen adherence and, together with hospitalization, are physiological stressors that precipitate decline and negatively impact the disease trajectory.

- Given the ageing population, there is need to adapt our approach to this patient group from one which is focused on single diseases to a care model which is proactive, coordinated and person-centred.

PD were on five or more repeat prescriptions [10] and had higher polypharmacy than controls even when controlling for the number of comorbidities. Prescribing cascades can result from clinicians misinterpreting an adverse drug effect as a new condition, for which another medication is then prescribed, which worsens polypharmacy (Fig. 1) [37].

The anticholinergic effects of drugs, which can be quantified using tools such as the anticholinergic burden calculator (http://www.acbcalc.com/), are under-recognised, potentially harmful and commonly prescribed in PD in the community [16] and hospital $[38,39]$. PD drugs including amantadine and monoamine oxidase inhibitors have anticholinergic effects [40] but drugs not used for PD motor symptoms, such as tricyclic antidepressants and drugs for overactive bladder, account for most of the anticholinergic burden in PD patients [38]. Critically, in patients with early PD, greater than six month exposure to anticholinergic PD drugs increases the risk of developing dementia relative to non-exposed individuals $(\mathrm{aHR}=1.23,95 \%$ CI: $1.10-1.32, p<0.001)[41]$ and high anticholinergic cumulative dose increases risk of dementia in PD [42].

Hospitalization of people with PD can precipitate motor deterioration [43] in part due to drug 
errors, which are common amongst PD inpatients and increase length of stay [44]. Development of delirium, commonly encountered during hospital admissions, may modify the disease trajectory [45]. Risk of delirium is likely high in PD [45] as a result of susceptibility to acute insult, age and PD-related cognitive and physical vulnerability [46]. As well as being a potential precipitant of adverse outcomes in this vulnerable group, increasing number and duration of hospital admissions appears to precede care home admission and may represent a period of crisis [47]. Amongst a prevalent UK population of people with idiopathic $\mathrm{PD}, 14 \%$ were living in a residential or nursing home and these individuals were older, with later stage disease, poorer cognitive function and worse functional status, likely indicative of frailty and multimorbidity [47]. The gravity of these negative outcomes provides a strong rationale to better identify frailty and/or multimorbidity and target intervention appropriately.

\section{CONCLUSION}

The heterogeneity of PD has long necessitated careful management [48]. Ageing invariably adds a cumulative complexity from multimorbidity encompassing PD, and frailty. Synergistic interactions between frailty and multimorbidity drive expenditure [49] in community and hospital [50] and pose a significant threat to the well-being of older patients and caregivers, who describe their experience of managing multimorbidity as 'overwhelming, draining and complicated' [51]. They feel 'split into pieces' by a system which fails to see them as a whole person and are burdened by the constant need to schedule pills and appointments [51] as well as symptom fluctuation and fatigue [48] which further erode coping capacity and contribute to the cycle of cumulative complexity [3]. The Comprehensive Geriatric Assessment (CGA), a diagnostic process undertaken by members of the multidisciplinary team, with close patient and caregiver involvement, looks beyond a single disease model in order to gain a multidimensional perspective on an individual's needs, taking into account frailty and multimorbidity [52]. Within PD care, this can then be used to develop an individualised and holistic care plan, which includes disease-specific expertise where necessary.

Active screening for and identification of frailty is important for several reasons. Firstly, frailty status can be used to inform decision-making around appropriate care, recognising that, amongst people living with frailty, hospital admission can have negative effects, including worsening of cognition and physical frailty. The requirement for patients to have good cognition to be a candidate for advanced therapies implicitly takes into account frailty status, and this may be a better guide to suitability for advanced therapies than eligibility criteria which apply an arbitrary upper age limit of 70 years [53]. In addition, frailty is considered to be a dynamic condition in which individuals may transition to an improved, as well as more advanced, frailty state [14]. It may also be possible to prevent, delay or reverse sarcopenia [15]. Therefore, early intervention for those on the trajectory towards sarcopenia and/or frailty has the potential to improve outcomes for individuals with PD.

There is an urgent need to address the limitations of current disease-centric care and research [54]. Recognition and management of complexity is likely to improve quality of life and prognosis for patients [55]. A more biopsychosocial model of care [56] that comprises regular, comprehensive patient assessment by an integrated team; case management; personalised care plans, prioritising patients' goals and preferences; self-management support for patients [57] is advocated, feasible and successful [58]. Future research studies should seek to enrol patients who are representative of the population living with $\mathrm{PD}$, multimorbidity and frailty to make findings generalisable to this prevalent, yet under-represented group. This approach to care, coupled with an inclusive research agenda, will undoubtedly improve the quality of life of people with $\mathrm{PD}$.

\section{ACKNOWLEDGMENTS}

EJH and ET receive salary support from the Gatsby Foundation.

\section{CONFLICT OF INTEREST}

ET has no conflict of interest to report. EJH has received research funding from the National Institute of Health Research (NIHR), Parkinson's-UK, the Gatsby Foundation and British Geriatric Society. She has received travel, consultancy, and honoraria from Profile, Bial, Abbvie, Luye, and Ever pharma. 


\section{REFERENCES}

[1] de Lau LML, Breteler MMB (2006) Epidemiology of Parkinson's disease. Lancet Neurol 5, 525-35.

[2] Levy G (2007) The relationship of Parkinson disease with aging. Arch Neurol 64, 1242-1246.

[3] Shippee ND, Shah ND, May CR, Mair FS, Montori VM (2012) Cumulative complexity: A functional, patientcentered model of patient complexity can improve research and practice. J Clin Epidemiol 65, 1041-1051.

[4] Buckley M, O'Neill D (2015) Ageism in studies of rehabilitation in Parkinson's disease. J Am Geriatr Soc 63, 1470-1471.

[5] Macleod AD, Henery R, Nwajiugo PC, Scott NW, Caslake R, Counsell CE (2018) Age-related selection bias in Parkinson's disease research: Are we recruiting the right participants? Parkinsonism Relat Disord 55, 128-133.

[6] Huntley AL, Johnson R, Purdy S, Valderas JM, Salisbury C (2012) Measures of multimorbidity and morbidity burden for use in primary care and community settings: A systematic review and guide. Ann Fam Med 10, 134-141.

[7] Macleod AD, Goddard H, Counsell CE (2016) Comorbidity burden in Parkinson's disease: Comparison with controls and its influence on prognosis. Parkinsonism Relat Disord 28, 124-129.

[8] Gravell R, Duncan GW, Khoo TK, Burn DJ, Sayer AA, Barker RA, Lawson RA, Yarnall AJ (2018) Multimorbidity predicts quality of life but not motor severity in early Parkinson's disease. J Parkinsons Dis 8, 511-515.

[9] Santos García D, Suárez Castro E, Expósito I, de Deus T, Tuñas C, Aneiros A, López Fernández M, Núñez Arias D, Bermúdez Torres M (2017) Comorbid conditions associated with Parkinson's disease: A longitudinal and comparative study with Alzheimer disease and control subjects. J Neurol Sci 373, 210-215.

[10] McLean G, Hindle J V, Guthrie B, Mercer SW (2017) Co-morbidity and polypharmacy in Parkinson's disease: Insights from a large Scottish primary care database. $B M C$ Neurol 17, 1-8.

[11] Johnston MC, Crilly M, Black C, Prescott GJ, Mercer SW (2019) Defining and measuring multimorbidity: A systematic review of systematic reviews. Eur J Public Health 29, 182-189.

[12] Cesari M, Pérez-Zepeda MU, Marzetti E (2017) Frailty and multimorbidity: Different ways of thinking about geriatrics. J Am Med Dir Assoc 18, 361-364.

[13] Ryan A, Wallace E, O'Hara P, Smith SM (2015) Multimorbidity and functional decline in community-dwelling adults: A systematic review. Health Qual Life Outcomes 13, 168.

[14] Hoogendijk EO, Afilalo J, Ensrud KE, Kowal P, Onder G, Fried LP (2019) Frailty: Implications for clinical practice and public health. Lancet 394, 1365-1375.

[15] Cruz-Jentoft AJ, Bahat G, Bauer J, Boirie Y, Bruyère O, Cederholm T, Cooper C, Landi F, Rolland Y, Sayer AA, Schneider SM, Sieber CC, Topinkova E, Vandewoude M, Visser M, Zamboni M, Bautmans I, Baeyens JP, Cesari M, Cherubini A, Kanis J, Maggio M, Martin F, Michel JP, Pitkala K, Reginster JY, Rizzoli R, Sánchez-Rodríguez D, Schols J (2019) Sarcopenia: Revised European consensus on definition and diagnosis. Age Ageing 48, $16-31$

[16] Weintraub D, Caspell-Garcia C, Simuni T, Cho HR, Coffey CS, Aarsland D, Alcalay RN, Barrett MJ, Chahine LM, Eberling J, Espay AJ, Hamilton J, Hawkins KA, Leverenz J, Litvan I, Richard I, Rosenthal LS, Siderowf A,
York M, Parkinson's Progression Markers Initiative (2020) Neuropsychiatric symptoms and cognitive abilities over the initial quinquennium of Parkinson disease. Ann Clin Transl Neurol 7, 449-461.

[17] Lethbridge L, Johnston GM, Turnbull G (2013) Comorbidities of persons dying of Parkinson's disease. Prog Palliat Care 21, 140-145.

[18] Brettschneider C, Leicht H, Bickel H, Dahlhaus A, Fuchs A, Gensichen J, Maier W, Riedel-Heller S, Schäfer I, Schön G, Weyerer S, Wiese B, van den Bussche H, Scherer M, König H-H (2013) Relative impact of multimorbid chronic conditions on health-related quality of life - results from the MultiCare Cohort Study. PLoS One 8, e66742.

[19] Formiga F, Ferrer A, Sanz H, Marengoni A, Alburquerque J, Pujol R (2013) Patterns of comorbidity and multimorbidity in the oldest old: The Octabaix study. Eur J Intern Med 24, 40-44.

[20] Potashkin J, Huang X, Becker C, Chen H, Foltynie T, Marras C (2020) Understanding the links between cardiovascular disease and Parkinson's disease. Mov Disord 35, 55-74

[21] Hong CT, Huang YH, Liu HY, Chiou HY, Chan L, Chien LN (2016) Newly diagnosed anemia increases risk of Parkinson's disease: A population-based cohort study. Sci Rep $\mathbf{6}$, $1-7$.

[22] Smith N, Brennan L, Gaunt DM, Ben-Shlomo Y, Henderson E (2019) Frailty in Parkinson's disease: A systematic review. J Parkinsons Dis 9, 517-524.

[23] Peball M, Mahlknecht P, Werkmann M, Marini K, Murr F, Herzmann H, Stockner H, De Marzi R, Heim B, Djamshidian A, Willeit P, Willeit J, Kiechl S, Valent D, Krismer F, Wenning GK, Nocker M, Mair K, Poewe W, Seppi K (2019) Prevalence and associated factors of sarcopenia and frailty in Parkinson's disease: A cross-sectional study. Gerontology 65, 216-228.

[24] The association between frailty and Parkinson's in the ReSPOnD trial - MS abstracts. 1090. 24th September 2019.

[25] Firat Ozer F, Akin S, Gultekin M, Erturk zararsiz G, Ekinci Soylu A (2019) Frailty in Patients with Parkinson's Disease: Associations with Disability and Timed Up and Go. Arch Neuropsychiatry, 1-8.

[26] Burt JR, Godin J, Filion J, Montero-Odasso M, Rockwood K, Andrew MK, Camicioli R (2019) Frailty prevalence in the COMPASS-ND study of neurodegenerative disorders. Can Geriatr J 22, 205-212.

[27] Torsney K, Romero-Ortuno R (2018) The Clinical Frailty Scale predicts inpatient mortality in older hospitalised patients with idiopathic Parkinson's disease. J R Coll Physicians Edinb 48, 103-107.

[28] Calderón-Larrañaga A, Vetrano DL, Ferrucci L, Mercer SW, Marengoni A, Onder G, Eriksdotter M, Fratiglioni L (2019) Multimorbidity and functional impairment-bidirectional interplay, synergistic effects and common pathways. $J$ Intern Med 285, 255-271.

[29] Peball M, Mahlknecht P, Werkmann M, Marini K, Murr F, Herzmann H, Stockner H, De Marzi R, Heim B, Djamshidian A, Willeit P, Willeit J, Kiechl S, Valent D, Krismer F, Wenning GK, Nocker M, Mair K, Poewe W, Seppi K (2019) Prevalence and associated factors of sarcopenia and frailty in Parkinson's disease: A cross-sectional study. Gerontology 65, 216-228.

[30] Vetrano DL, Pisciotta MS, Laudisio A, Lo Monaco MR, Onder G, Brandi V, Fusco D, Di Capua B, Ricciardi D, Bernabei R, Zuccalà G (2018) Sarcopenia in Parkinson disease: Comparison of different criteria and association with disease severity. J Am Med Dir Assoc 19, 523-527. 
[31] Sheard JM, Ash S, Silburn PA, Kerr GK (2011) Prevalence of malnutrition in Parkinson's disease: A systematic review. Nutr Rev 69, 520-532.

[32] Peball M, Mahlknecht P, Werkmann M, Marini K, Murr F, Herzmann H, Stockner H, de Marzi R, Heim B, Djamshidian A, Willeit P, Willeit J, Kiechl S, Nocker M, Mair K, Poewe W, Seppi K (2018) Clinical characteristics of frailty in Parkinson's disease. Mov Disord 33, S729.

[33] Torsney KM, Romero-Ortuno R (2018) The clinical frailty scale predicts inpatient mortality in older hospitalised patients with idiopathic Parkinson's disease. J R Coll Physicians Edinb 48, 103-107.

[34] Smith N, Brennan L, Gaunt DM, Ben-Shlomo Y, Henderson E (2019) Frailty in Parkinson's disease: A systematic review. J Parkinsons Dis 9, 517-524.

[35] National Institute for Health and Care Excellence (2016) National Institute for Clinical Excellence. Multimorbidity: Clinical assessment and management, NICE, United Kingdom.

[36] Müller-Rebstein S, Trenkwalder C, Ebentheuer J, Oertel WH, Culmsee C, Höglinger GU (2017) Drug safety analysis in a real-life cohort of Parkinson's disease patients with polypharmacy. CNS Drugs 31, 1093-1102.

[37] Piggott KL, Mehta N, Wong CL, Rochon PA (2020) Using a clinical process map to identify prescribing cascades in your patient. BMJ 261, m261.

[38] Lertxundi U, Isla A, Solinis MA, Domingo-Echaburu S, Hernandez R, Peral-Aguirregoitia J, Medrano J (2015) Anticholinergic burden in Parkinson's disease inpatients. Eur J Clin Pharmacol 71, 1271-1277.

[39] Crispo JAG, Willis AW, Thibault DP, Fortin Y, Hays HD, McNair DS, Bjerre LM, Kohen DE, Perezlloret S, Mattison DR, Krewski D (2016) Associations between anticholinergic burden and adverse health outcomes in Parkinson disease. PLoS One 11, 1-15.

[40] Klietz M, Greten S, Wegner F, Höglinger GU (2019) Safety and tolerability of pharmacotherapies for Parkinson's disease in geriatric patients. Drugs Aging 36, 511-530.

[41] Hong CT, Chan L, Wu D, Chen WT, Chien LN (2019) Antiparkinsonism anticholinergics increase dementia risk in patients with Parkinson's disease. Parkinsonism Relat Disord 65, 224-229.

[42] Sheu JJ, Tsai MT, Erickson SR, Wu CH (2019) Association between anticholinergic medication use and risk of dementia among patients with Parkinson's disease. Pharmacotherapy 39, 798-808.

[43] Gerlach OHH, Broen MPG, van Domburg PHMF, Vermeij AJ, Weber WEJ (2012) Deterioration of Parkinson's disease during hospitalization: Survey of 684 patients. BMC Neurol 12, 2-7.

[44] Lertxundi U, Isla A, Solinís MÁ, Echaburu SD, Hernandez R, Peral-Aguirregoitia J, Medrano J, García-Moncó JC (2017) Medication errors in Parkinson's disease inpatients in the Basque Country. Parkinsonism Relat Disord 36, 57-62.

[45] Lawson RA, McDonald C, Burn DJ (2019) Defining delirium in idiopathic Parkinson's disease: A systematic review. Parkinsonism Relat Disord 64, 29-39.

[46] Vardy ERLC, Teodorczuk A, Yarnall AJ (2015) Review of delirium in patients with Parkinson's disease. J Neurol 262, 2401-2410.

[47] Klaptocz J, Gray WK, Marwood S, Agarwal M, Ziegler J, Webb Z, Prabhakar M, Hand A, Oates L, McDonald C,
Walker RW (2019) The pattern of hospital admissions prior to care home placement in people with Parkinson's disease: Evidence of a period of crisis for patients and carers. J Aging Health 31, 1616-1630.

[48] Ryden LE, Lewis SJG (2019) Parkinson's disease in the era of personalised medicine: One size does not fit all. Drugs Aging 36, 103-113.

[49] Bhattacharjee S, Sambamoorthi U (2013) Co-occurring chronic conditions and healthcare expenditures associated with Parkinson's disease: A propensity score matched analysis. Parkinsonism Relat Disord 19, 746-750.

[50] Wang X, Zeng F, Jin WS, Zhu C, Wang QH, Bu X Le, Luo HB, Zou HQ, Pu J, Zhou ZH, Cui XP, Wang QS, Shi XQ, Han W, Wu Q, Chen HS, Lin H, Zhang LL, Zhang M, Lian Y, Xu ZQ, Zhou HD, Zhang T, Wang YJ (2017) Comorbidity burden of patients with Parkinson's disease and Parkinsonism between 2003 and 2012: A multicentre, nationwide, retrospective study in China. Sci Rep 7, 1-6.

[51] Ploeg J, Matthew-Maich N, Fraser K, Dufour S, McAiney C, Kaasalainen S, Markle-Reid M, Upshur R, Cleghorn L, Emili A (2017) Managing multiple chronic conditions in the community: A Canadian qualitative study of the experiences of older adults, family caregivers and healthcare providers. BMC Geriatr 17, 1-15.

[52] Radder DLM, de Vries NM, Riksen NP, Diamond SJ, Gross D, Gold DR, Heesakkers J, Henderson E, Hommel ALAJ, Lennaerts HH, Busch J, Dorsey RE, Andrejack J, Bloem BR (2019) Multidisciplinary care for people with Parkinson's disease: The new kids on the block! Expert Rev Neurother 19, 145-157.

[53] Antonini A, Stoessl AJ, Kleinman LS, Skalicky AM, Marshall TS, Sail KR, Onuk K, Odin PLA (2018) Developing consensus among movement disorder specialists on clinical indicators for identification and management of advanced Parkinson's disease: A multi-country Delphipanel approach. Curr Med Res Opin 34, 2063-2073.

[54] Bloem B, Henderson E, Dorsey R, Okun M, Okubadejo N, Chan P, Andrejack J, Darweesh S, Munneke M (2020) Integrated and patient-centred management of Parkinson's disease: A network model for reshaping chronic neurological care. Lancet Neurol. Press.

[55] Tangianu F, Gnerre P, Colombo F, Frediani R, Pinna G, Berti F, Mathieu G, La Regina M, Orlandini F, Mazzone A, Canale C, Borioni D, Nardi R (2018) Could clustering of comorbidities be useful for better defining the internal medicine patients' complexity? Ital J Med 12, 137-144.

[56] Gibson G (2017) What can the treatment of Parkinson's disease learn from dementia care; applying a bio-psychosocial approach to Parkinson's disease. Int J Older People Nurs 12, 1-8.

[57] Palmer K, Marengoni A, Forjaz MJ, Jureviciene E, Laatikainen T, Mammarella F, Muth C, Navickas R, PradosTorres A, Rijken M, Rothe U, Souchet L, Valderas J, Vontetsianos T, Zaletel J, Onder G (2018) Multimorbidity care model: Recommendations from the consensus meeting of the Joint Action on Chronic Diseases and Promoting Healthy Ageing across the Life Cycle (JA-CHRODIS). Health Policy (New York) 122, 4-11.

[58] Fleisher J, Barbosa W, Sweeney MM, Oyler SE, Lemen AC, Fazl A, Ko M, Meisel T, Friede N, Dacpano G, Gilbert RM, Di Rocco A, Chodosh J (2018) Interdisciplinary home visits for individuals with advanced Parkinson's disease and related disorders. J Am Geriatr Soc 66, 1226-1232. 